\title{
Tendances concernant l'incidence du cancer, la mortalité par cancer et la survie au cancer au Canada entre 1970 et 2007
}

\author{
L. Kachuri, M.H.P. (1); P. De, Ph. D. (1, 2); L. F. Ellison, M. Sc. (3); R. Semenciw, M. Sc. (4); Comité consultatif des \\ statistiques sur le cancer
}

Cet article a fait l'objet d'une évaluation par les pairs.

Résumé

Introduction : La surveillance des tendances concernant le cancer peut aider à évaluer les progrès réalisés dans la lutte contre le cancer ainsi qu'à renforcer les activités de prévention. La présente étude, fondée sur les données des bases de données nationales, consiste en un examen des tendances à long terme relatives à certains cancers au Canada.

Méthodologie : Nous avons examiné les changements annuels dans les tendances relatives aux taux d'incidence et de mortalité normalisés selon l'âge observés entre 1970 et 2007 selon le sexe pour tous les cancers combinés, pour les quatre cancers les plus courants (cancer de la prostate, cancer du sein, cancer du poumon, cancer colorectal) et pour les cancers dont les tendances ont été marquées par les changements les plus importants au cours des dernières années. Le rapport de survie relative à cinq ans pour les années 1992 à 2007 a également été calculé.

Résultats : Pendant la période visée par l'étude, les taux d'incidence de l'ensemble des cas de cancer primitif combinés ont augmenté de 0,9\% par année chez les hommes et de $0,8 \%$ par année chez les femmes. Les taux concernant le mélanome, le cancer de la thyroïde, le cancer du foie, le cancer de la prostate, le cancer du rein, le cancer colorectal, le cancer du poumon, le cancer du sein et le cancer de la vessie ont augmenté à des rythmes variables, et les taux concernant le cancer du larynx, le cancer de la bouche, le cancer de l'estomac et le cancer du col de l'utérus ont diminué. Pour l'ensemble des cancers combinés et pour la plupart des cancers examinés, à l'exception du mélanome et du cancer du poumon chez les femmes, les taux de mortalité ont diminué significativement. Les taux de survie qui ont le plus augmenté sont ceux du cancer de la prostate, du cancer du foie, du cancer colorectal et du cancer du rein. Bien que les tendances globales concernant les taux de mortalité et la survie indiquent que des progrès notables ont été réalisés dans la lutte contre le cancer, la tendance à la hausse des taux d'incidence de certains cancers soulignent la nécessité de poursuivre les efforts dans le domaine de la prévention.

Mots-clés :surveillance du cancer, incidence, mortalité, survie, facteurs de risque

\section{Introduction}

Au début de 2007, près de 750000 Canadiens vivants avaient été touchés par le cancer au cours des 10 années précédentes ${ }^{1}$. Le cancer est la principale cause de décès au Canada ${ }^{2}$, et $82 \%$ des décès causés par le cancer surviennent chez des personnes âgées de 60 ans et plus ${ }^{1}$. D’ici 2036, environ 10,9 millions de Canadiens seront âgés de 65 ans ou plus ${ }^{3}$, ce qui donnera lieu à une augmentation du nombre de nouveaux cas de cancers et sera à l'origine d'une forte demande en soins de santé liés au cancer.

Un examen des tendances passées peut nous aider à prévoir les tendances futures liées au cancer et à évaluer les progrès réalisés dans la lutte contre cette maladie, et ainsi permettre aux professionnels de la santé publique de renforcer les activités existantes dans les domaines de la prévention et de la lutte contre le cancer.

Cette analyse consiste en un examen des tendances à long terme concernant l'ensemble des cancers combinés, les quatre cancers les plus courants au Canada (cancer de la prostate, cancer du sein chez la femme, cancer du poumon et cancer colorectal) et les cancers dont les tendances en matière d'incidence ou de mortalité ont été marquées par les changements les plus importants au cours des dix dernières années (cancer de l'estomac, cancer du foie, cancer de la thyroïde, cancer du larynx, mélanome, cancer de la vessie, cancer du rein et cancer du col de l'utérus). À notre connaissance, il s'agit de l'examen le plus à jour et le plus exhaustif des tendances à long terme concernant le cancer au Canada. À ce titre, il peut être utilisé pour établir des comparaisons avec les tendances signalées dans d'autres pays. Plus important encore, les tendances ont été analysées dans le contexte des principaux facteurs de risque de cancer et des comportements associés en matière de santé afin d'offrir un point de vue sur les déterminants possibles de la maladie.

Rattachement des auteurs :

1. École Dalla Lana de santé publique, Université de Toronto, Toronto (Ontario), Canada

2. Politiques de lutte contre le cancer, Société canadienne du cancer, Toronto (Ontario), Canada

3. Division des statistiques sur la santé, Statistique Canada, Ottawa (Ontario), Canada

4. Centre de prévention et de contrôle des maladies chroniques, Agence de la santé publique du Canada, Ottawa (Ontario), Canada

Correspondance : Prithwish De, Société canadienne du cancer, 55, avenue St. Clair Ouest, bureau 300, Toronto (Ontario) M4V 2Y7; tél. : 416-934-5335; téléc. : 416-961-4189;

courriel : prithwish.de@cancer.ca 


\section{Méthodologie}

\section{Sources de données}

Nous avons utilisé les données sur l'incidence du cancer de 1992 à 2007 disponibles dans la version de juillet 2010 du Registre canadien du cancer, une base de données sur la population axée sur la personne ${ }^{4}$. Les données de la période antérieure, de 1970 à 1991, sont tirées du Système national de déclaration des cas de cancer, une base de données axée sur les tumeurs créée en $1969^{5}$. Les données sur la mortalité sont tirées de la Base canadienne de données sur l'état civil Décès de Statistique Canada. Enfin, les estimations de la population viennent du Compendium des estimations démographiques de 2010 de Statistique Canada ${ }^{6}$.

Nous avons créé un fichier contenant des données sur les cas de cancer invasif, pour tous les âges, et sur les cas de cancer in situ de la vessie (à l'exception des cas de la province de l'Ontario) à l'aide des règles de codage des tumeurs primitives multiples du Centre international de recherche sur le cancer ${ }^{7}$. Les cas de cancer ont été classifiés selon les critères de la $3^{\mathrm{e}}$ édition de la Classification internationale des maladies pour l'oncologie (CIM-O) ${ }^{8}$. Les définitions des groupes de cancers sont fournies ailleurs ${ }^{1}$. Pour les décès attribuables au cancer, les causes sousjacentes des décès ont été sélectionnées selon les classes énoncées dans la Classification internationale des maladies, version $10(\mathrm{CIM}-10)^{9}$.

\section{Analyse statistique}

Nous avons calculé les taux selon l'âge pour chaque année, puis les avons ajustés en fonction de la répartition par âge de la population canadienne de 1991 afin d'obtenir les taux d'incidence (TINA) et de mortalité (TMNA) normalisés selon l'âge. Nous avons analysé les tendances à court et à long terme en calculant la variation annuelle en pourcentage (VAP) et la variation annuelle moyenne en pourcentage (VAMP) dans les taux, respectivement, à l'aide de la version 3.5.1 du logiciel Joinpoint ${ }^{10}$. Ce logiciel utilise la régression linéaire pour modéliser la variation des TINA et des TMNA sur l'échelle logarithmique. D’autres méthodes sont utilisables, par exemple l'ajustement polynomial des données, mais Joinpoint caractérise les tendances de manière plus succincte en faisant correspondre la pente de chaque segment à une variation moyenne en pourcentage ${ }^{11}$. Il fallait disposer d'au moins cinq années de données avant et après un point de variation pour dégager une nouvelle tendance. Les modèles ont été testés à l'aide de la méthode de permutation de Monte Carlo (significatif à $p=0,05$ ). Chaque variation statistiquement significative de la tendance est décrite ici comme étant « décroissante » ou « à la baisse » ou, au contraire, « croissante ».

Les analyses de la survie relative ont été fondées sur un algorithme accessible au public $^{12}$ que nous avons légèrement adapté. Cette étude a porté sur tous les individus atteints de cancer primitif âgés de 15 à 99 ans au moment du diagnostic. Le suivi de mortalité jusqu'au 31 décembre 2007 a été réalisé par le couplage des enregistrements du Registre canadien du cancer et de la Base canadienne de données sur l'état civil - Décès de Statistique Canada, et d'après les renseignements fournis par les registres provinciaux/territoriaux du cancer. Les données du Québec ont été exclues parce que la méthode utilisée dans cette province pour établir la date à laquelle le diagnostic de cancer a été posé diffère de celle utilisée dans les autres provinces ${ }^{13,14}$ et en raison de questions liées à la détermination du statut vital exact des cas diagnostiqués au Québec dans le Registre canadien du cancer. Nous avons effectué des estimations du rapport de survie relative (RSR) à cinq ans à l'aide de la méthode des cohortes pour 1992 à 1994, 1996 à 1998 et 2000 à 2002, et à l'aide de la méthode fondée sur la période pour 2005 à 2007. Comme nous ne disposions pas de données plus récentes, nous avons supposé que les données sur la survie pour la période de 2005 à 2007 (utilisées pour établir la survie relative) étaient les mêmes que celles de 2000 à 2002. De plus amples renseignements sur la méthode utilisée pour établir la survie sont fournis ailleurs $^{15}$.

\section{Résultats}

En 2007, 85430 nouveaux cas de cancer et 36569 décès attribuables au cancer ont été déclarés chez les hommes, et 78099 nouveaux cas et 33026 décès ont été déclarés chez les femmes. Ensemble, les cancers les plus fréquemment diagnostiqués (cancer de la prostate, cancer du sein chez la femme, cancer colorectal et cancer du poumon) représentaient respectivement $55 \%$ chez les hommes et $52 \%$ chez les femmes de tous les nouveaux diagnostics de cancer, et $50 \%$ chez les hommes et 51 $\%$ chez les femmes des décès causés par le cancer.

\section{Tendances concernant l'incidence et la mortalité tous cancers combinés}

\section{Tous cancers combinés}

Le tableau 1 présente la VAP et la VAMP des taux d'incidence du cancer. Les TINA pour l'ensemble des cancers de 1970 et de 2007 étaient plus élevés chez les hommes (1970 : 330,4 pour 100 000; 2007 : 463,2 pour 100 000) que chez les femmes (1970 : 272,0 pour 100 000; 2007 : 362,3 pour 100 000). Pendant la période visée par l'étude, ce taux a augmenté à un rythme moyen de 0,9 \% par année chez les hommes et de 0,8 \% par année chez les femmes.

La VAP et la VAMP des taux de mortalité par cancer sont présentées dans le tableau 2. Comme dans le cas de l'incidence, les TMNA pour tous les cancers combinés étaient plus élevés chez les hommes (1970 : 228,4 pour 100 000; 2007: 200,1 pour 100 000) que chez les femmes (1970: 152,1 pour 100 000; 2007 : 141,2 pour 100000 ), mais ils ont diminué de 0,3\% par année chez les hommes et de $0,2 \%$ par année chez les femmes sur la période de l'étude.

\section{Tendances spécifiques}

Entre 1970 et 2007, on a observé chez les hommes une tendance générale à la hausse des taux d'incidence (figure 1) du mélanome (VAMP : 3,7 \%), du cancer de la thyroïde $(3,6 \%)$, du cancer du foie $(3,5 \%)$, du cancer de la prostate $(2,2 \%)$, du cancer du rein $(1,8 \%)$, du cancer colorectal $(0,6 \%)$ et du cancer de la vessie $(0,4 \%)$, mais une tendance à la baisse en 


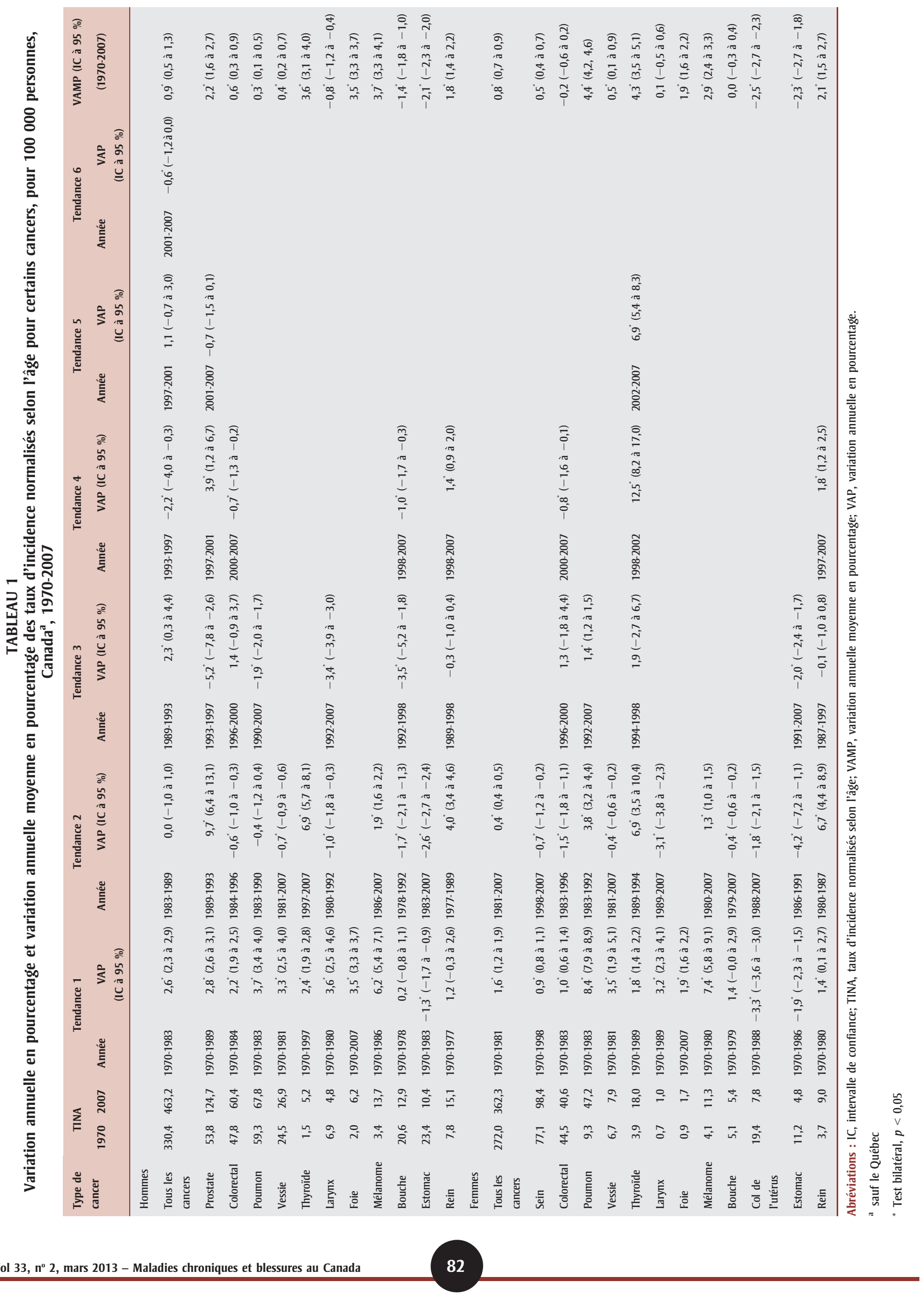




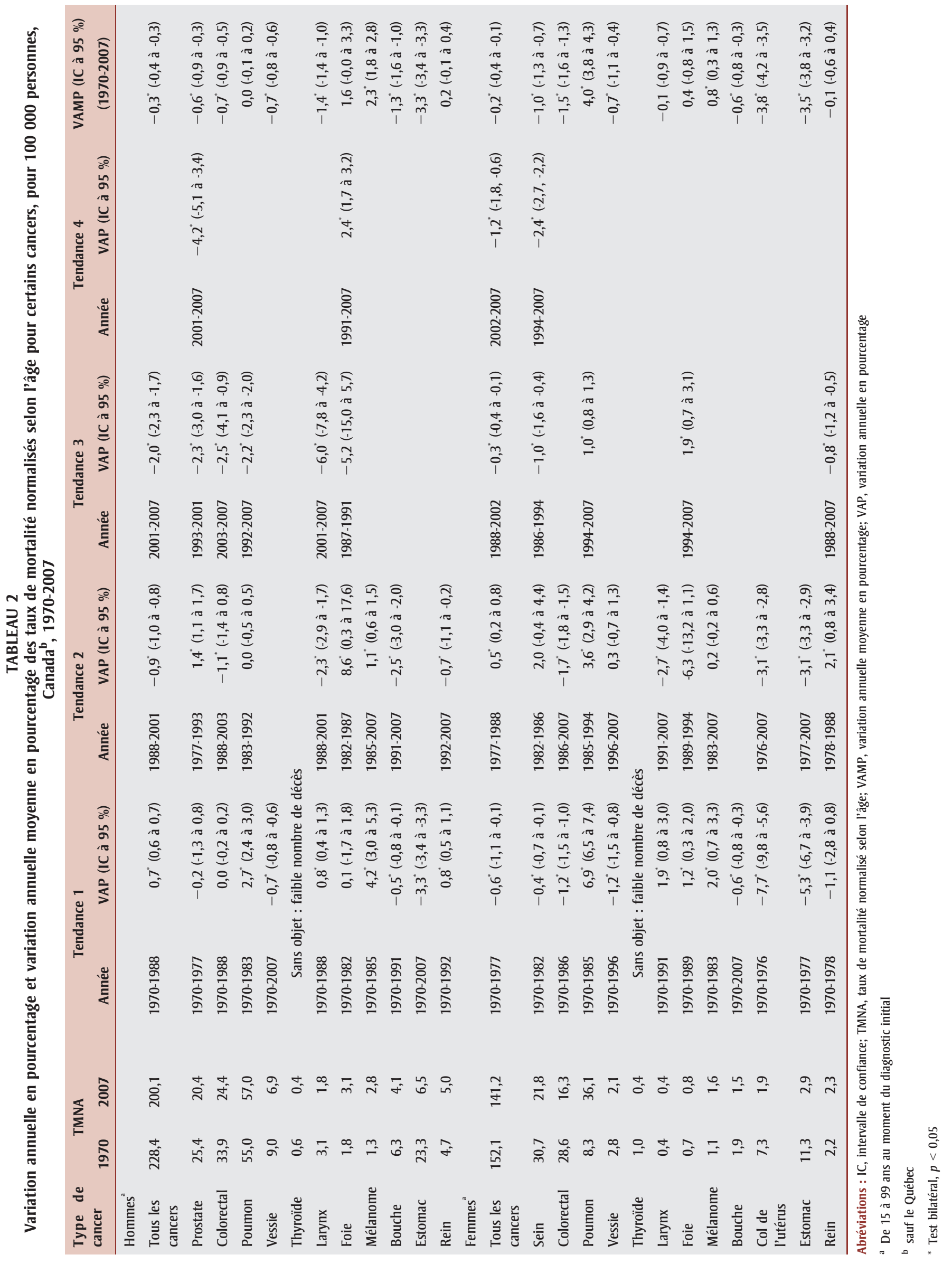


FIGURE 1

Variation annuelle moyenne en pourcentage des taux d'incidence normalisés selon l'âge pour certains cancers, hommes et femmes, Canada sauf le Québec, 1970-2007

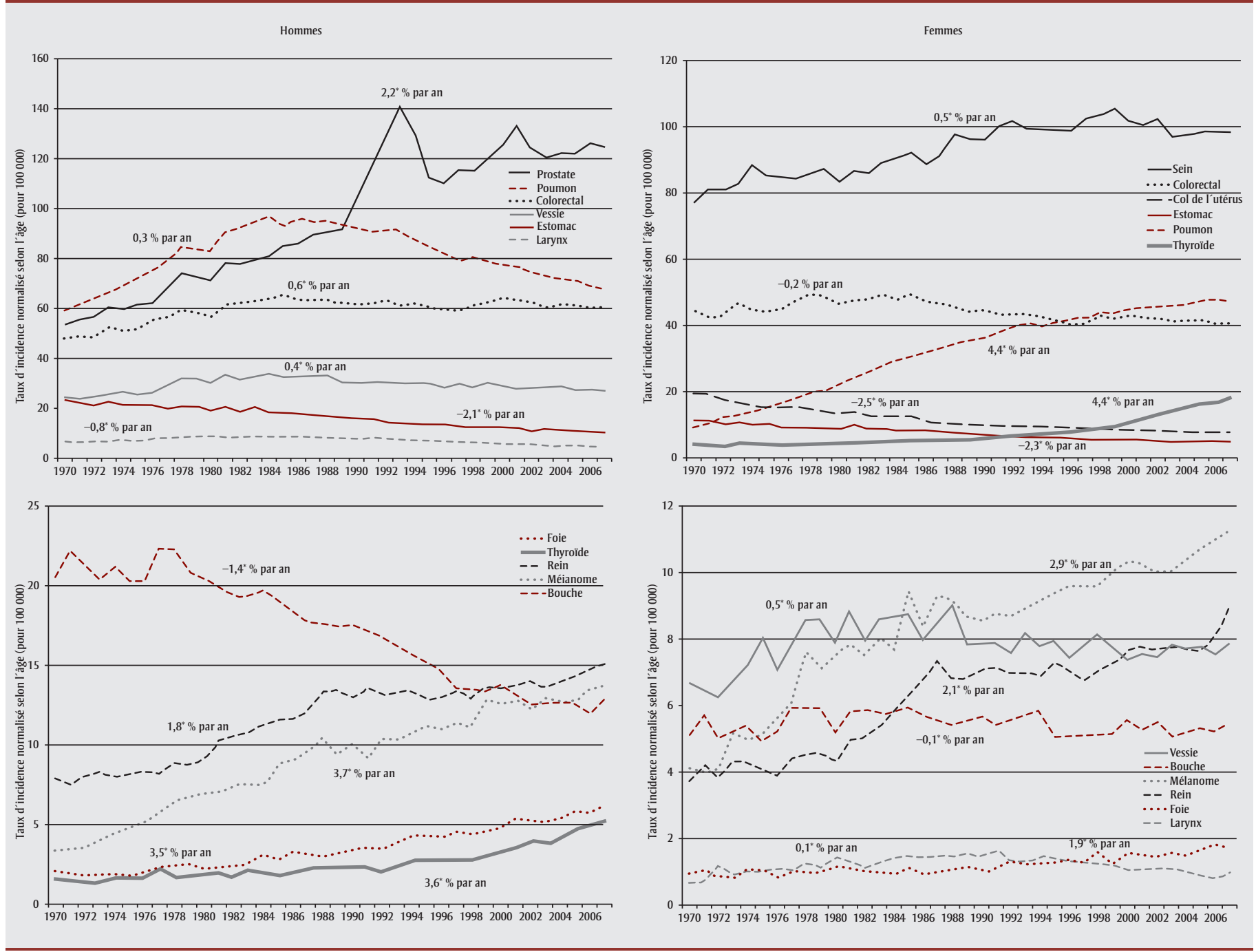

Remarque : Analyses Joinpoint, jusqu'à 5 points de retournement, basées sur des taux ajustés selon l'âge (pour 100000 personnes)

${ }^{*}$ Test bilatéral, $p<0,05$

ce qui concerne le cancer du larynx $(0,8 \%)$, le cancer de la bouche $(1,4 \%)$ et le cancer de l'estomac $(2,1 \%)$. Chez les femmes, on a observé une augmentation des taux d'incidence du cancer de la thyroïde (VAMP : 4,4\%), du cancer du poumon $(4,4 \%)$, du mélanome $(2,9 \%)$, du cancer du rein $(2,1 \%)$, du cancer du foie $(1,9 \%)$, du cancer du sein $(0,5 \%)$ et du cancer de la vessie $(0,5 \%)$, mais une diminution de la VAMP dans le cas du cancer du col de l'utérus $(2,5 \%)$ et du cancer de l'estomac (2,3\%).

Pour la plupart des cancers, les taux de mortalité enregistrés entre 1970 et 2007 étaient caractérisés par des diminutions statistiquement significatives (figure 2), sauf dans le cas du cancer du poumon chez les femmes (VAMP : 4,0 \%) et du mélanome (VAMP : 2,3 \% chez les hommes, $0,8 \%$ chez les femmes), pour lesquels des augmentations ont été observées.

Il vaut la peine de mettre en évidence les tendances concernant certains cancers. Par exemple, le taux d'incidence du cancer de la prostate a atteint deux points culminants, en 1993 et en 2001 (tableau 1 et figure 1 ). Après le premier pic, le taux d'incidence a diminué (VAP : 5,2 \%) jusqu'en 1997, après quoi il a augmenté de 3,9 \% par année pour atteindre un deuxième pic en 2001, suivi d'une période de baisse non significative. Nous avons observé une seule période d'augmentation du taux de mortalité par ce cancer, entre 1977 et 1993, suivie d'une baisse continue qui s'est davantage intensifiée depuis 2001 (tableau 2).

Le taux d'incidence du cancer du poumon a augmenté de 3,7\% par année chez les hommes entre 1970 et 1983 . Cette hausse a été suivie d'une période de variation non significative jusqu'en 1990, date à laquelle le taux d'incidence a commencé à baisser (tableau 1). Chez les femmes, on observe depuis 1970 une augmentation du taux d'incidence, mais elle a ralenti pour passer 
FIGURE 2

Variation annuelle moyenne en pourcentage des taux de mortalité normalisés selon l'âge pour certains cancers, hommes et femmes, Canada sauf le Québec, 1970-2007

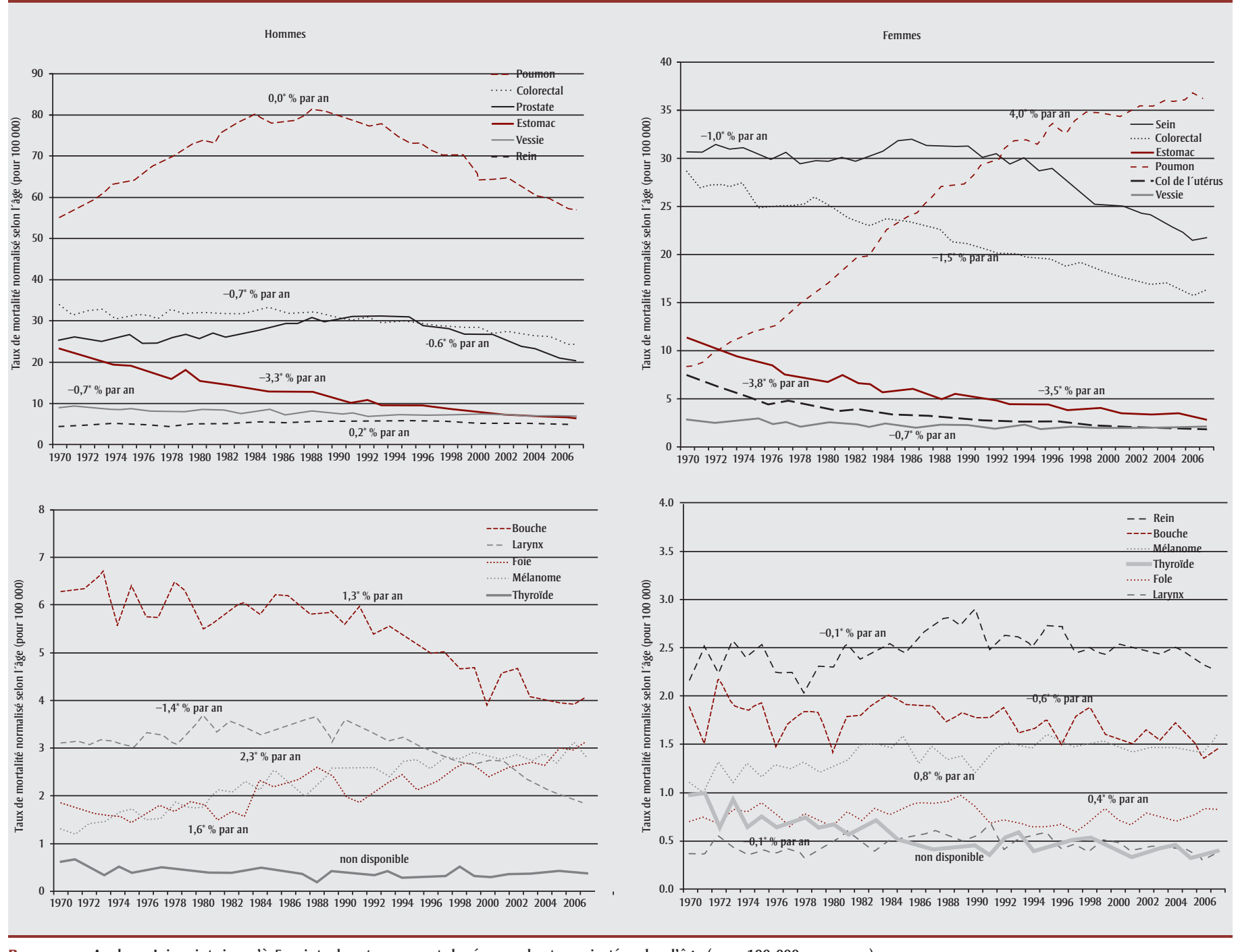

Remarque : Analyses Joinpoint, jusqu'à 5 points de retournement, basées sur des taux ajustés selon l'âge (pour 100000 personnes)

${ }^{*}$ Test bilatéral, $p<0,05$

de 8,4 \% par année (1970-1983) à 3,8 \% par année (1983-1992) pour finalement atteindre 1,4 \% par année (1992-2007). Chez les hommes, les taux de mortalité par cancer du poumon ont suivi une trajectoire similaire à celle des taux d'incidence : les taux ont augmenté (2,7 \% par an) jusqu'en 1983, sont demeurés stables (0,0 \% par an) de 1983 à 1992, puis ont commencé à baisser à un rythme annuel de 2,2 \% (tableau 2). Au contraire, les taux de mortalité par cancer du poumon enregistrés chez les femmes continuent d'augmenter depuis 1970 : le rythme annuel d'augmentation a cependant ralenti, de 6,9 \% (1970-1985) à 3,6\%
(1985-1994) pour finalement atteindre 1,0\% (1994-2007).

Les taux d'incidence du cancer du larynx ont augmenté de 1970 à 1980 chez les hommes (VAP : 3,6 \%) et jusqu'en 1989 chez les femmes (3,2 \% par année). Chez les hommes, les taux d'incidence ont baissé de 1,0 \% par année de 1980 à 1992, après quoi la baisse s'est accélérée à 3,4 \% par année. Chez les femmes, on observe une baisse annuelle de 3,1 \% du taux d'incidence depuis 1989. Les taux de mortalité ont augmenté de 1970 à 1988 chez les hommes $(0,8 \%$ par année) et jusqu'en $1991 \mathrm{chez}$ les femmes (1,9\% par année), mais ces hausses ont été suivies de baisses significatives chez les deux sexes.

Les taux d'incidence du cancer de la vessie ont augmenté de 1970 à 1981 (hommes: 3,3 \% par an; femmes : 3,5\% par an), puis la tendance s'est inversée en 1981 et les taux d'incidence ont commencé à diminuer (hommes : $0,7 \%$ par an; femmes: 0,4 \% par an). Les taux de mortalité ont connu dans le même temps une diminution significative sur l'ensemble de la période pour les hommes $(0,7 \%$ par an) et de 1970 à 1996 pour les femmes (1,2\% par an). 
Le taux d'incidence global du cancer du rein a connu chez les hommes deux périodes d'augmentation, de 1977 à 1989 et de 1998 à 2007. Nous avons observé deux périodes d'augmentation similaires chez les femmes, de 1980 à 1987 et de 1997 à 2007. Quant au taux de mortalité, il a augmenté de 1970 à 1992 chez les hommes puis a diminué, tandis que chez les femmes il augmenté de 1978 à 1988 puis a diminué.

Le taux d'incidence du cancer de la thyroïde a augmenté de façon constante pour les deux sexes depuis 1970. Chez les hommes, il a augmenté à un rythme de 2,4 \% par année entre 1970 et 1997, puis a augmenté à un rythme accéléré de 6,9 \% par année jusqu'en 2007. Plus particulièrement, le taux d'incidence chez les femmes a varié de 1,8\% par année entre 1970 et 1989 , de 6,9 \% par année entre 1989 et 1994, de 12,5\% par année entre 1998 et 2002 et, plus récemment (2002-2007), de 6,9\% par année. Les taux de mortalité par cancer de la thyroïde étaient trop faibles pour permettre la réalisation d'une analyse Joinpoint.

\section{Tendances concernant la survie}

Entre 1992 et 1994 et entre 2005 et 2007, les RSR à cinq ans normalisés selon l'âge pour tous les cancers combinés ont augmenté de 6,8 points de pourcentage et atteint $62 \%$ (tableau 3). Les taux de survie ont davantage augmenté chez les hommes que chez les femmes $(8,5$ points de pourcentage contre 5,0$)$ pendant cette période, ce qui a donné lieu à une diminution considérable de l'écart précédent.

L'augmentation du RSR à cinq ans a considérablement varié selon le type de cancer. Les plus importantes augmentations d'approximativement 8 à 10 points de pourcentage ont touché le cancer de la prostate, le cancer du foie, le cancer colorectal et le cancer du rein. De légères augmentations de 2 à 3 points de pourcentage ont été observées dans le cas du cancer du poumon, du cancer du larynx, du cancer du col de l'utérus et du cancer de la bouche. Aucune augmentation apparente n'a été observée dans le cas du cancer de la vessie pendant la période

TABLEAU 3

Rapports de survie relative à cinq ans normalisés selon l'âge pour certains cancers, par période, Canada sauf le Québec, 1992-2007

\begin{tabular}{|c|c|c|c|c|c|}
\hline \multirow[t]{2}{*}{ Type de cancer } & \multicolumn{4}{|c|}{ RSR $^{b}$ en \% (IC à 95 \%) } & \multirow{2}{*}{$\begin{array}{c}\text { Variation }^{c} \\
1992-1994 \text { à } \\
2005-2007\end{array}$} \\
\hline & 1992-1994 & 1996-1998 & $2000-2002$ & 2005-2007 & \\
\hline Tous les cancers & 56 (55 à 56) & 57 (57 à 57) & 60 (60 à 61) & 62 (62 à 63) & 6,8 \\
\hline Hommes $^{\mathrm{a}}$ & 54 (54 à 54) & 55 (55 à 56) & 60 (60 à 60) & 62 (62 à 63) & 8,5 \\
\hline Femmes $^{\mathrm{a}}$ & 57 (56 à 57) & 58 (58 à 59) & 60 (60 à 60) & 62 (62 à 63) & 5,0 \\
\hline Prostate & 87 (86 à 87) & 90 (89 à 90) & 94 (94 à 95) & 96 (96 à 97) & 9,8 \\
\hline Sein (femme) & 82 (82 à 83) & 85 (85 à 86) & 87 (86 à 87) & 88 (87 à 88$)$ & 5,6 \\
\hline Colorectal & 56 (55 à 56) & 58 (57 à 59) & 61 (60 à 61) & 64 (64 à 65) & 8,6 \\
\hline Poumon & 14 (13 à 14) & 15 (14 à 15) & 15 (15 à 15) & 16 (16 à 17) & 2,6 \\
\hline Vessie & 73 (72 à 74) & 71 (70 à 72) & 71 (70 à 72) & 72 (71 à 73) & $-0,3$ \\
\hline Thyroïde & 93 (92 à 94) & 94 (93 à 95) & 96 (96 à 97) & 98 (97 à 98) & 4,9 \\
\hline Larynx & $62(60$ à 64) & 63 (61 à 65) & 62 (59 à 64) & 64 (61 à 66) & 1,9 \\
\hline Foie & 10 (8 à 11) & 12 (11 à 14) & 17 (15 à 18) & 18 (17 à 20) & 8,7 \\
\hline Mélanome & 84 (83 à 86) & 87 (86 à 87) & 89 (88 à 90) & 89 (89 à 90) & 4,9 \\
\hline Bouche & 60 (59 à 61) & 59 (58 à 61) & 61 (59 à 62) & 62 (61 à 64) & 2,3 \\
\hline Col de l'utérus & 70 (68 à 71) & 70 (69 à 72) & 73 (71 à 75) & 72 (70 à 73) & 2,2 \\
\hline Estomac & 19 (18 à 21) & 22 (20 à 23) & 22 (21 à 23) & 25 (23 à 26) & 5,1 \\
\hline Rein & 60 (58 à 61) & 62 (60 à 63) & 64 (63 à 65) & 67 (66 à 69) & 7,7 \\
\hline
\end{tabular}

Abréviations: IC, intervalle de confiance; RSR, rapport de survie relative.

a Personnes âgées de 15 à 99 ans au moment du diagnostic initial

b Résultats obtenus à l'aide de la méthode des cohortes (1992-1994, 1996-1998 et 2000-2002) et de la méthode fondée sur la période (2005-2007)

c Différence absolue en points de pourcentage

visée par l'étude. En ce qui concerne l'augmentation de la survie, les disparités entre les sexes avantageaient les femmes (données non présentées) et touchaient le cancer de la bouche $(3,7 \%$ chez les femmes contre $1,1 \%$ chez les hommes), le cancer du larynx (4,1\% contre 1,5\%), le cancer du poumon (3,5 \% contre 1,3\%) et le cancer de l'estomac $(6,3 \%$ contre $4,5 \%)$.

\section{Analyse}

Au cours de la période de près de 40 ans analysée (1970-2007), les taux d'incidence de tous les cancers combinés ont augmenté significativement tant chez les hommes que chez les femmes du Canada. Bien que les taux se soient stabilisés chez les hommes depuis 1993, le taux global d'incidence chez les femmes ne semble avoir commencé à se stabiliser que récemment. Ces tendances globales ont été largement déterminées par les trois cancers les plus courants chez les hommes (c.-à-d. cancer du poumon, cancer de la prostate et cancer colorectal) et chez les femmes (c.-à-d. cancer du sein, cancer du poumon et cancer colorectal).

Les taux de mortalité par cancer chez les deux sexes ont atteint un point culminant en 1988 et ont depuis baissé, en grande partie en raison d'une diminution des taux de mortalité associés aux quatre principales causes de mortalité par cancer (c.-à-d. cancer du poumon, cancer colorectal, cancer de la prostate et cancer du sein). L'augmentation des RSR à cinq ans depuis la période 1992-1994 en ce qui concerne tous les cancers combinés et quelques cancers spécifiques laisse croire à des améliorations dans les traitements et la détection précoce de certains cancers ainsi qu'à des progrès dans les soins médicaux de soutien et généraux.

\section{Tendances pour les principaux cancers}

\section{Cancer de la prostate}

Mis à part l'âge, on dispose de peu de données sur les facteurs de risque du 
cancer de la prostate. Bien que les androgènes jouent un rôle critique dans la croissance des cellules cancéreuses de la prostate, on ne sait pas avec certitude si des taux élevés d'androgènes peuvent constituer un facteur d'initiation du cancer ${ }^{16}$. Un lien avec l'activité physique a été suggéré ${ }^{17}$, mais les données probantes demeurent peu concluantes $^{18,19}$. L'obésité n'est que faiblement associée au développement du cancer de la prostate, mais certains suggèrent qu'elle pourrait accroître le risque de décès et de métastases ${ }^{20-22}$.

Malgré l'incertitude quant aux bienfaits et aux risques associés au test de dépistage du cancer de la prostate par dosage de l'antigène prostatique spécifique (APS), ce test est largement utilisé ${ }^{23}$. Selon les enquêtes nationales sur la santé, la proportion d'hommes âgés de 35 ans et plus ayant déjà subi un test de dosage de l'APS s'élevait à $53,8 \%$ en $2008^{24}$. Deux essais randomisés menés récemment n’ont pas permis de confirmer que le test de dosage de l'APS était un outil viable pour le dépistage dans la population et la réduction du nombre de décès dus au cancer de la prostate ${ }^{25,26}$, et il n'est actuellement pas recommandé au Canada comme test de dépistage dans la population. Néanmoins, le taux d'incidence du cancer de la prostate au Canada a fortement augmenté à la suite de l'introduction du test de dosage de l'APS en 1988. Le taux d'incidence a atteint un point culminant en 1993, puis un autre en 2001. Ce deuxième point culminant pourrait s'expliquer par la publicité faite cette année-là lorsque le ministre fédéral de la Santé de l'époque a révélé qu'il avait reçu un diagnostic de cancer de la prostate.

Les taux de mortalité par cancer de la prostate au Canada ont diminué depuis 1995, revenant en 2007 aux taux qui prévalaient avant 1970 . On croit que la détection précoce du cancer de la prostate grâce aux vastes activités de dépistage aurait contribué au ralentissement de la tendance concernant la mortalité aux États-Unis ${ }^{27}$, et certains suggèrent qu'un phénomène similaire est responsable des tendances concernant la mortalité et la survie observées au Canada ${ }^{28}$. Le fait que le RSR à cinq ans ait gagné presque 10 points de pourcentage depuis la période de 1992 à 1994 pourrait également s'expliquer, dans une certaine mesure, par la plus grande accessibilité à des traitements hormonaux efficaces pour les personnes aux stades précoce et avancé de la maladie vers le milieu des années $1980^{29}$, suivie de l'introduction de surveillance vigilante et de progrès réalisés dans les domaine de la radiothérapie combinée et de l'hormonothérapie pour le cancer de la prostate, qui ont eu lieu dans les années $1990^{30}$.

\section{Cancer du sein}

Au Canada, le taux d'incidence du cancer du sein chez les femmes a augmenté régulièrement à un rythme de 0,9\% par année entre 1970 et 1998, période après laquelle il a commencé à diminuer à un rythme de 0,7 \% par année. Les tendances concernant l'incidence du cancer du sein reflètent vraisemblablement les changements à long terme dans les facteurs hormonaux (p. ex. âge précoce des premières règles, âge tardif de la ménopause, allaitement au sein, utilisation de contraceptifs oraux, recours au traitement hormonal substitutif) et la participation accrue au dépistage par mammographie, en particulier dans les années $1980^{31}$. Le premier programme provincial de dépistage du cancer du sein a été mis en œuvre en 1988 au Canada et, en 1998, des programmes avaient été mis sur pied dans les dix provinces canadiennes ${ }^{32}$. Bien que tous les programmes provinciaux offrent des tests de dépistage par mammographie aux femmes âgées de 50 à 69 ans, certains sont également accessibles aux femmes dans la quarantaine et à celles de plus de 69 ans $^{32}$.

Les enquêtes nationales sur la santé montrent que la proportion de femmes ménopausées âgées de 50 à 69 ans qui déclarent avoir subi une mammographie au cours des deux années précédentes a augmenté, passant de 40,5\% en 1990 à $72,5 \%$ en $2008^{32}$. La brève diminution du taux d'incidence du cancer du sein entre 1998 et 2005 pourrait être due à l'épuisement des cas prévalents non diagnostiqués grâce au dépistage comme à une diminution du risque de cancer du sein due au fait que certaines femmes ménopausées évitent le traitement hormonal substitutif depuis les rapports de la Women's Health Initiative et les enquêtes antérieures qui ont mis en évidence les risques associés à ce traitement ${ }^{33}$.

Bien que la consommation d'alcool et l'obésité chez les femmes ménopausées puissent accroître le risque de cancer du $\operatorname{sein}^{34,35}$ et que l'activité physique puisse réduire ce risque ${ }^{36}$, l'incidence de ces facteurs dans le contexte canadien reste à éclaircir.

Le taux de mortalité par cancer du sein a commencé à baisser en 1986 à un rythme de 1,0\% par an, puis a augmenté à raison de 2,4 \% par an après 1994 . La diminution du taux de mortalité et l'augmentation de la survie résultaient vraisemblablement du recours accru à la mammographie opportuniste avant la mise en place des programmes de dépistage provinciaux, du recours accru à l'hormonothérapie et à la chimiothérapie adjuvante et $^{37,38}$ et du virage de la pratique clinique vers la chirurgie conservatrice du sein et la tumorectomie $\mathrm{e}^{39,40}$.

\section{Cancer du poumon}

Le tabagisme est un facteur causal du développement du cancer du poumon, du cancer de la bouche et du cancer du larynx, entre autres ${ }^{41}$. Les effets du tabagisme sur l'incidence du cancer du poumon sont seulement observés après une période de latence d'environ $25 \mathrm{ans}^{42}$. La prévalence du tabagisme au Canada a beaucoup diminué entre 1965 et 2007, passant de $61 \%$ à $20 \%$ chez les hommes et de $38 \%$ à $18 \%$ chez les femmes de 15 ans et plus ${ }^{43}$. Après avoir atteint un point culminant en 1965, l'usage du tabac a chuté après que les effets négatifs du tabagisme eurent été largement publicisés dans un rapport du directeur du service de santé publique des États-Unis ${ }^{44}$. Cela a entraîné une baisse de l'incidence du cancer du poumon chez les hommes après le sommet atteint en 1983, et une baisse du taux de mortalité chez les hommes après le sommet atteint en 1988. En 2007, le taux d'incidence du cancer du poumon chez les hommes était à peu près redescendu au même niveau qu'en 1970.

Par contre, le taux de mortalité par cancer du poumon chez les femmes a continué 
d'augmenter, quoiqu'à un rythme plus lent depuis le milieu des années 2000. Les taux de tabagisme chez les femmes ont commencé à diminuer environ 15 ans après les taux observés chez les hommes, se stabilisant entre $37 \%$ et $39 \%$ jusqu'en $1979^{43}$. Bien que l'on continue d'observer une tendance à la hausse en ce qui concerne le taux d'incidence du cancer du poumon et le taux de mortalité attribuable à ce dernier chez les femmes canadiennes, des données encourageantes publiées par les É.-U. montrent que le taux de décès lié au cancer du poumon chez les femmes dans ce pays diminue après avoir atteint un plateau ${ }^{45}$.

\section{Cancer colorectal}

Le cancer colorectal est associé à plusieurs risques modifiables, comme l'obésité, la sédentarité, la consommation de viandes rouges et transformées ainsi que le tabagisme $^{46}$. La prévalence de l'obésité (c.-à-d. indice de masse corporelle $\geq 30$ ) chez les adultes canadiens a augmenté pendant 30 ans jusqu'en 2007-2009, passant de $13,8 \%$ à $23,9 \%{ }^{47,48}$. La prévalence était plus élevée chez les femmes (15,9\%) que chez les hommes (11,5 \%) en 1978-1979, mais cette tendance s'est inversée, si bien qu'une proportion légèrement plus élevée d'hommes (24,2 \%) que de femmes $(23,6 \%)$ étaient considérés comme obèses en 2007-2009 ${ }^{47,48}$.

Le taux d'incidence du cancer colorectal chez les hommes est revenu au niveau observé au début des années 1980, tandis que le taux chez les femmes est maintenant plus faible que celui qui était observé dans les années 1970. Chez les hommes, la baisse du taux de décès a commencé en 1988, tandis que chez les femmes, la baisse de ce taux a été amorcée avant 1970 et se poursuit. Les différences dans ces tendances suggèrent divers facteurs de risque. De l'avis de certains, le fait que les femmes aient davantage eu recours au traitement hormonal substitutif avant le début des années 2000 pourrait avoir contribué à la diminution du risque de cancer du côlon chez les personnes de ce sexe $e^{34,49}$.

Chez les deux sexes, les taux de décès associés au cancer colorectal ont commencé à diminuer avant la participation accrue au dépistage par l'intermédiaire des programmes globaux mis en œuvre dans l'ensemble des provinces canadiennes au cours des six dernières années. La recherche de sang occulte dans les selles chez les personnes de 50 ans et plus présentant un risque moyen ${ }^{50}$ et la coloscopie dans le cas des personnes à risque élevé sont les principales méthodes utilisées pour la détection précoce et l'excision des polypes précancéreux ${ }^{51}$ ainsi que pour la réduction de l'incidence du cancer colorectal et de la mortalité attribuable à ce dernier. Actuellement, le taux moyen de participation des personnes âgées de 50 à 74 ans aux programmes de dépistage dans les provinces est de $32,2 \%{ }^{52}$. Une plus grande participation au dépistage contribuera vraisemblablement à réduire davantage le taux d'incidence du cancer colorectal et le taux de mortalité par ce cancer au Canada.

\section{Cancers avec tendance à la hausse des taux d'incidence}

\section{Cancer de la thyroïde}

$\mathrm{Au}$ Canada, le cancer de la thyroïde fait partie des cancers qui ont connu la croissance la plus rapide au cours des dernières années ${ }^{1,53}$. La forte tendance à la hausse pourrait être due à l'utilisation accrue des technologies de diagnostic telles que l'aspiration à l'aiguille pour la détection des tumeurs infracliniques, au fait que l'exposition accrue au rayonnement ionisant à des fins diagnostiques puisse favoriser le développement de nouvelles tumeurs, ou à l'exposition accrue à un facteur de risque environne-

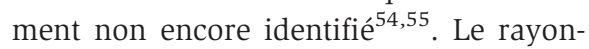
nement ionisant demeure le facteur de risque le plus connu du cancer de la thyroïde, mais de plus en plus de données probantes font ressortir le rôle possible des facteurs liés au poids et à l'appareil reproducteur de la femme, qui interviennent probablement tous les deux dans la carcinogenèse par la voie des hormones. Malgré le taux d'incidence croissant du cancer de la thyroïde, les taux de mortalité associés à ce cancer sont demeurés faibles et le RSR à cinq ans chez les deux sexes (98 \%) est le plus élevé de l'ensemble des principaux cancers.

\section{Cancer du foie}

Le type de cancer primitif du foie le plus courant, le carcinome hépatocellulaire, est associé à un faible taux de survie et à un taux de mortalité élevé. Entre 1970 et 2007, l'incidence du cancer du foie au Canada a augmenté plus rapidement chez les hommes (3,5 \% par année) que chez les femmes (1,9 \% par année). Les différences dans les taux d'incidence observés entre les deux sexes pourraient être dues à la répartition différente des facteurs de risque du cancer du foie, par exemple la surconsommation d'alcool (c.-à-d. au-delà des Directives de consommation d'alcool à faible risque ${ }^{56}$ ), le tabagisme et l'hépatite. Les estimations basées sur la population montrent que le taux de surconsommation d'alcool au Canada a augmenté entre 1989 et 2007, passant de $18,9 \%$ à $25 \%$ chez les hommes et de $7,2 \%$ à $9,6 \%$ chez les femmes $^{57,58}$.

Dans les pays développés, le fardeau lié au cancer du foie est principalement causé par l'infection chronique par l'hépatite $C$ plutôt qu'à l'infection au virus de l'hépatite B (VHB), dont la prévalence est plus élevée ailleurs dans le monde ${ }^{59}$. On a également émis l'hypothèse selon laquelle il existerait un lien entre l'obésité et le cancer du foie, qui pourrait être causé par la stéatose hépatique non alcoolique ${ }^{60}$. De telles associations soulignent la nécessité de se soucier de l'augmentation du taux d'obésité au Canada, qui est passé de $14 \%$ à la fin des années 1970 à $24 \%$ en 2007-2009 ${ }^{47,48}$.

\section{Mélanome}

Le rayonnement ultraviolet peut causer toutes les formes de cancer de la peau ${ }^{61}$. Bien que la hausse de l'incidence du mélanome au Canada puisse en partie s'expliquer par une meilleure détection $^{62,63}$, cette hausse découle plus vraisemblablement d'une exposition accrue aux rayons UV dans le cadre des loisirs, que ces rayons proviennent du soleil ou de dispositifs de bronzage artificiel. Selon l'Enquête nationale sur l'exposition au soleil de 2006, la prévalence du bronzage est d'environ $49 \%$ chez les femmes canadiennes et de $28 \%$ chez les hommes canadiens âgés de 16 à 24 ans $^{64}$. 
Des principaux cancers, le mélanome est le deuxième dont le taux de mortalité a le plus augmenté (après le cancer du foie chez les hommes et le cancer du poumon chez les femmes) depuis 1970. Demeuré globalement stable depuis 1983 chez les femmes, le taux de mortalité associé au mélanome a augmenté de 1,1 \% par an chez les hommes durant une période équivalente (1985-2007). Le plus faible RSR à cinq ans $^{65}$ et la proportion plus élevée de cas à un stade plus avancé chez les hommes ${ }^{66}$ font que le taux de mortalité est plus élevé chez eux que chez les femmes. Toutefois, on observe un ralentissement de la tendance à la hausse du taux de mortalité chez les hommes, peut-être en raison de l'augmentation de la survie grâce à la détection précoce et à l'amélioration des traitements du mélanome, en particulier l'exérèse chirurgicale $^{67}$.

\section{Rein}

L'augmentation du taux d'incidence du cancer du rein, bien que cela ne soit pas clair, pourrait s'expliquer par plusieurs changements, dont l'accessibilité à de nouvelles techniques de diagnostic ${ }^{68,69}$, ainsi que par l'augmentation de la prévalence de l'obésité et de l'hypertension, deux importants facteurs de risque ${ }^{70}$. En fait, au Canada, 55 \% des cas de cancer du rein chez les hommes et $27 \%$ des cas chez les femmes pourraient être attribuables au surpoids ou à l'obésité ${ }^{71}$.

\section{Cancers avec tendance à la baisse des taux d'incidence}

\section{Estomac, col de l'utérus, bouche, larynx, vessie}

Le tabagisme est un important facteur de risque des cancers de l'estomac, de la bouche, du larynx, de la vessie et du col de l'utérus. La tendance à la baisse de l'incidence de ces cancers et de la mortalité associée à ceux-ci peut en grande partie s'expliquer par les tendances relatives au tabagisme, qui a considérablement diminué après 1965 chez les hommes et après 1979 chez les femmes au Canada ${ }^{43}$. Les changements touchant les autres facteurs de risque ont également eu une influence. Par exemple, la diminution du taux de cancer de l'estomac depuis les années 1970 est attribuable aux améliorations apportées au régime alimentaire, notamment la consommation accrue de fruits et de légumes et la diminution de la consommation d'aliments en conserve contenant du sel ${ }^{72}$, et, plus récemment, à l'amélioration de la détection et du traitement de l'infection à Helicobacter pylori, l'un des principaux facteurs de risque du cancer de l'estomac ${ }^{73}$.

Pendant la période visée par l'étude, les taux observés au Canada de l'incidence du cancer du col de l'utérus et de la mortalité attribuable à ce cancer ont continué à diminuer en raison de l'utilisation généralisée du test de dépistage de Papanicolaou (Pap) mis sur le marché en $1949^{74}$. En association avec le test de Pap, l'administration aux femmes âgées de 9 à 26 ans d'un vaccin contre le virus du papillome humain (VPH) (approuvé au Canada en $2008^{75}$ ) devrait réduire davantage les taux d'incidence et de mortalité à long terme. Compte tenu du fait que le $\mathrm{VPH}$ est de plus en plus reconnu comme l'une des causes de certains cancers de la bouche, par exemple ceux qui apparaissent sur les amygdales et l'oropha$\operatorname{rynx}^{76,77}$, la vaccination contre le VPH pourrait également contribuer à influer les tendances futures au Canada.

\section{Limites}

Cette étude comporte plusieurs limites. Premièrement, nous avons tenté d'expliquer les tendances observées en matière de cancer en utilisant des données démographiques relatives à des facteurs de risques, facteurs largement transversaux et, pour la plupart, autodéclarés. Deuxièmement, en raison de la disponibilité des données, nous avons seulement pu prendre en considération un sousensemble de facteurs du style de vie modifiables ayant une incidence sur les taux de la maladie. De plus, nous avons seulement pris en compte les facteurs de risque modifiables pouvant, du point de vue étiologique, s'appliquer aux cancers apparaissant à l'âge adulte, mais non ceux ceux qui s'appliquent uniquement aux cancers de l'enfant et de l'adolescent. Troisièmement, les sources de données, les méthodes d'enregistrement des cas de cancer, ainsi que la complétude et l'exactitude des données utilisées pour élaborer les estimations de l'incidence peuvent varier à l'échelle du Canada ${ }^{1}$. De telles différences peuvent conduire à des sous-estimations et à des surestimations mineures des taux de la maladie, qui sont examinés plus en détail ailleurs ${ }^{1}$. Enfin, les estimations de survie relative pour 2005 à 2007 pourraient être exagérées, car nous avons dû les élaborer en utilisant les données sur la survie prévue d'une période antérieure. L'effet est probablement plus marqué dans le cas des cancers ayant une proportion plus élevée de sujets plus âgés, par exemple le cancer de la prostate.

\section{Conclusion}

Les tendances à la baisse en ce qui concerne les taux d'incidence de certains cancers et les taux de mortalité de la plupart des cancers au Canada montrent la réussite de diverses stratégies, en particulier le dépistage du cancer, la prévention par les changements de mode de vie et de comportement ainsi que les améliorations concernant l'hygiène environnementale. Malgré ces réalisations, il demeure important de renforcer les activités de prévention primaire, car plusieurs cancers continuent d'afficher des taux d'incidence à tendances stables ou croissantes.

\section{Remerciements}

Ces travaux ont été réalisés grâce aux contributions en nature de la Société canadienne du cancer, de l'Agence de la santé publique du Canada et de Statistique Canada. Les auteurs remercient le Comité directeur des statistiques sur le cancer pour son examen critique du manuscrit: Heather Chappell (Société canadienne du cancer), Dagny Dryer (Centre de traitement du cancer de l'Î.-P.-É.), Maureen MacIntyre (Action Cancer NouvelleÉcosse), Loraine Marrett (Action Cancer Ontario), Les Mery (Agence de la santé publique du Canada) et Hannah Weir (Centers for Disease Control and Prevention des É.-U.).

Le Registre canadien du cancer est tenu à jour par Statistique Canada. Il est constitué de données fournies par les registres provinciaux et territoriaux du cancer, que 
les auteurs tiennent à remercier pour leur collaboration.

\section{Références}

1. Société canadienne du cancer. Comité directeur des statistiques sur le cancer. Statistiques canadiennes sur le cancer, 2011. Toronto (Ont.) : Société canadienne du cancer; 2011.

2. Statistique Canada. Les principales causes de décès au Canada, 2009 [Internet]. Ottawa (Ont.) : Statistique Canada; 2012 [consulté le 19 septembre 2012]. (Statistique Canada, $\mathrm{n}^{\mathrm{o}}$ 84-215-XWF au catalogue). Consultable à partir de la page: http://www5.statcan.gc.ca/bsolc/olc-cel $/$ olc-cel catno $=84-215-\mathrm{x} \&$ lang $=$ fra

3. Statistique Canada. Projections démographiques pour le Canada, les provinces et les territoires, 2009 à 2036 [Internet]. Ottawa (Ont.) : Statistique Canada; 2010 [consulté le 5 août 2011]. (Statistique Canada, $n^{0}$ 91250-X au catalogue). Consultable à partir de la page : http://www.statcan.gc.ca/dailyquotidien/100526/dq100526b-fra.htm

4. Statistique Canada. Incidence du cancer au Canada, 2007 et 2008 [Internet]. Ottawa (Ont.) : Statistique Canada; 2010 [consulté le 5 août 2011]. (Statistique Canada, $n^{\circ} 82$ 231-X au catalogue). Consultable à partir de la page: http://www.statcan.gc.ca/pub/82231-x/82-231-x2009001-fra.htm

5. Band, Pierre R. Développement du registre canadien du cancer: incidence du cancer au Canada et dans les régions canadiennes, 1969-1988. Ottawa (Ont.) : Conseil canadien des registres du cancer/Santé et bienêtre social Canada/Statistique Canada; 1993.

6. Statistique Canada. Compendium des estimations démographiques, 2010. Tableau 051-0001 de CANSIM, diffusé le 29 septembre 2010. Ottawa (Ont.) : ministère de l'Industrie; 2010.

7. Parkin DM, Chen VW, Ferlay J, Galceran J, Storm HH, Whelan SL. Comparabilité et contrôle de qualité dans l'enregistrement des cancers. Lyon (FR) : Centre international de recherche sur le cancer/ Organisation mondiale de la Santé; 1994.
8. Fritz AG, Percy C, Jack A, Shanmugarathan K, Sobin L, Parkin DM et collab. (dir.). Classification internationale des maladies: oncologie (CIM-O), $3^{\mathrm{e}}$ édition. Genève (Suisse) : Organisation mondiale de la Santé; 2008.

9. Classification statistique internationale des maladies et des problèmes de santé connexes (CIM-10), $10^{\mathrm{e}}$ révision. Genève (Suisse) : Organisation mondiale de la Santé; 2008.

10. Joinpoint regression program, version 3.5.1 [logiciel]. Rockville (MD) : Statistical Research and Applications Branch, National Cancer Institute; 2011.

11. Kim HJ, Fay MP, Feuer EJ, Midthune DN Permutation tests for joinpoint regression with applications to cancer rates. Stat Med. 2000;19(3):335-51.

12. Dickman PW. Estimating and modelling relative survival using SAS [Internet]. [Stockholm (SE)]: [éditeur inconnu]; [consulté le 4 janvier 2010]. Consultable à partir de la page : http://www.pauldickman.com /rsmodel/sas_colon

13. Ellison LF, Gibbons L; Groupe d'analyse de la survie au cancer au Canada. Taux relatifs de survie à cinq ans: cancers de la prostate, du sein, du côlon et du rectum, et du poumon. Rapports sur la santé. 2001;13(1):25-38.

14. Louchini R. Beaupré, M. La survie au cancer pour les nouveaux cas déclarés au Québec en 1992. Montréal (Québec) : Institut national de santé publique, Direction du développement et des programmes, Unité connaissance-surveillance; 2002.

15. Statistiques sur la survie au cancer, 1992 à 2002 [Internet]. Ottawa (Ont.): Statistique Canada; 2011 [consulté le 5 juillet 2011]. (Statistique Canada, $n^{\circ}$ 82-226-X au catalogue). Consultable à partir de la page : http://www.statcan.gc.ca/pub/82-226-x /82-226-x2011001-fra.htm

16. Wang D, Tindall DJ. Androgen action during prostate carcinogenesis. Methods Mol Biol. 2011;776:25-44.

17. Vainio H, Bianchini $F$, (dir.) Weight control and physical activity.Lyon (FR) : International Agency for Research on Cancer; 2002. (IARC handbook of cancer prevention 6)
18. Pan SY, DesMeules M. Energy intake, physical activity, energy balance, and cancer : epidemiologic evidence. Methods Mol Biol. 2009;472:191-215.

19. Friedenreich CM, Neilson HK, Lynch BM. State of the epidemiological evidence on physical activity and cancer prevention. Eur J Cancer. 2010;46(14):2593-604.

20. MacInnis RJ, English DR. Body size and composition and prostate cancer risk : systematic review and meta-regression analysis. Cancer Causes Control. 2006; 17(8):989-1003.

21. Giovannucci E, Liu Y, Platz EA, Stampfer MJ, Willett WC. Risk factors for prostate cancer incidence and progression in the health professionals follow-up study. Int $\mathrm{J}$ Cancer. 2007;121(7):1571-8.

22. Gong Z, Agalliu I, Lin DW, Stanford JL, Kristal AR. Obesity is associated with increased risks of prostate cancer metastasis and death after initial cancer diagnosis in middle-aged men. Cancer. 2007;109(6): 1192-202.

23. Levy I. Prostate cancer : the epidemiologic perspective. Can J Oncol. 1994;4 Suppl 1: 4-7.

24. Statistique Canada. Enquêtes sur la santé dans les collectivités canadiennes - 2007 Cycle 4.1 [fichier informatique]. Ottawa (Ont.) : Statistique Canada, Division de la statistique de la santé; 2007.

25. Andriole GL, Crawford ED, Grubb RL 3rd, Buys SS, Chia D, Church TR et collab. Mortality results from a randomized prostate-cancer screening trial. N Engl J Med. 2009;360(13):1310-9.

26. Schröder FH, Hugosson J, Roobol MJ, Tammela TL, Ciatto S, Nelen V et collab. Screening and prostate-cancer mortality in a randomized European study. N Engl J Med. 2009;360(13):1320-8.

27. Hankey BF, Feuer EJ, Clegg LX, Hayes RB, Legler JM, Prorok PC et collab. Cancer surveillance series : interpreting trends in prostate cancer--part I : evidence of the effects of screening in recent prostate cancer incidence, mortality, and survival rates. J Natl Cancer Inst. 1999;91(12): 1017-24. 
28. Nam RK, Klotz LH. Trends in prostate cancer screening : Canada. Dans: Ankerst DP, Tangen CM, Thompson IM, (dir.), Current clinical urology : prostate cancer screening, $2^{\mathrm{e}}$ éd. New York (US) : Humana Press; 2009. p. 23-33.

29. Meng MV, Grossfeld GD, Sadetsky N, Mehta SS, Lubeck DP, Carroll PR. Contemporary patterns of androgen deprivation therapy use for newly diagnosed prostate cancer. Urology. 2002;60(3 Suppl 1):7-11.

30. Kupelian PA, Buchsbaum JC, Elshaikh MA, Reddy CA, Klein EA. Improvement in relapse-free survival throughout the PSA era in patients with localized prostate cancer treated with definitive radiotherapy: year of treatment an independent predictor of outcome. Int J Radiat Oncol Biol Phys. 2003;57(3):629-34.

31. Holford TR, Cronin KA, Mariotto AB, Feuer EJ. Changing patterns in breast cancer incidence trends. J Natl Cancer Inst Monogr.2006;(36):19-25.

32. Shields M, Wilkins K. Le point sur la mammographie au Canada. Rapports sur la santé. 2009;20(3):1-14.

33. De P, Neutel CI, Olivotto I, Morrison H. Breast cancer incidence and hormone replacement therapy in Canada. J Natl Cancer Inst. 2010;102(19):1489-95.

34. Reeves GK, Pirie K, Beral V, Green J, Spencer E, Bull D. Cancer incidence and mortality in relation to body mass index in the Million Women Study : cohort study. BMJ. 2007;335(7630):1134.

35. Alcohol consumption and ethyl carbamate. Lyon (FR): International Agency for Research on Cancer; 2010. (IARC monographs on the evaluation of carcinogenic risks to humans 96).

36. Friedenreich $\mathrm{C}$. The role of physical activity in breast cancer etiology. Semin Oncol. 2010;37(3):297-302.

37. Harlan LC, Abrams J, Warren JL, Clegg L, Stevens J, Ballard-Barbash R. Adjuvant therapy for breast cancer: practice patterns of community physicians. J Clin Oncol. 2002;20(7):1809-17.
38. Mariotto A, Feuer EJ, Harlan LC, Wun LM, Johnson KA, Abrams J. Trends in use of adjuvant multi-agent chemotherapy and tamoxifen for breast cancer in the United States: 1975-1999. J Natl Cancer Inst. 2002;94(21):1626-34.

39. Edwards BK, Brown ML, Wingo PA, Howe HL, Ward E, Ries LA et collab. Annual report to the nation on the status of cancer, 1975-2002, featuring population-based trends in cancer treatment. J Natl Cancer Inst. 2005;97(19):1407-27.

40. NIH consensus conference. Treatment of early-stage breast cancer. JAMA. 1991; 265(3):391-5.

41. Tobacco smoke and involuntary smoking. Summary of data reported and evaluation. Lyon (FR) : International Agency for Research on Cancer; 2004. (IARC monographs on the evaluation of carcinogenic risks to humans 83 )

42. Kleinsmith LJ. Principles of cancer biology, $1^{\mathrm{e}}$ éd. San Francisco (CA) : Benjamin Cummings; 2005.

43. Reid J, Hammond D. Tobacco use in Canada: patterns and trends, 2011 edition. Waterloo (Ont.) : Propel Centre for Population Health Impact, University of Waterloo; 2011.

44. The Surgeon General's Advisory Committee on Smoking and Health. Smoking and health: report of the Advisory Committee to the Surgeon General of the Public Health Service. Washington (DC) : US Department of Health, Education and Welfare, 1964. (Public Health Service Publication n 1103)

45. Kohler BA, Ward E, McCarthy BJ, Schymura MJ, Ries LA, Eheman C et collab. Annual report to the nation on the status of cancer, 1975-2007, featuring tumors of the brain and other nervous system. J Natl Cancer Inst. 2011;103(9):714-36.

46. Continuous Update Project interim report summary. Food, nutrition, physical activity, and the prevention of cancer, a global perspective: colorectal cancer [Internet]. Washington (US) : World Cancer Research Fund/American Institute for Cancer Research; 2007 [consulté le 19 sept. 2012]. Consultable à la page: http://www .dietandcancerreport.org/cup/current_ progress/colorectal_cancer.php
47. Tjepkema M. Obésité chez les adultes. Rapports sur la santé. 2006;17(3):9-26.

48. Statistique Canada. Enquête canadienne sur les mesures de la santé: tableaux de données du cycle 1, 2007 à 2009. Ottawa (Ont.) : Statistique Canada; 2010.

49. Newcomb PA, Zheng Y, Chia VM, Morimoto LM, Doria-Rose VP, Templeton A et collab. Estrogen plus progestin use, microsatellite instability, and the risk of colorectal cancer in women. Cancer Res. 2007;67(15):7534-9.

50. Mandel JS, Church TR, Bond JH, Ederer F, Geisser MS, Mongin SJ et collab. The effect of fecal occult-blood screening on the incidence of colorectal cancer. N Engl J Med. 2000;343(22):1603-7.

51. Schatzkin A, Freedman LS, Dawsey SM, Lanza E. Interpreting precursor studies : what polyp trials tell us about large-bowel cancer. J Natl Cancer Inst. 1994;86(14): 1053-7.

52. Partenariat canadien contre le cancer. Rapport de 2011 sur le rendement du système de lutte contre le cancer. Toronto (Ont.) : Partenariat canadien contre le cancer; 2010 .

53. Liu S, Semenciw R, Ugnat AM, Mao Y. Increasing thyroid cancer incidence in Canada, 1970-1996: time trends and ageperiod-cohort effects. $\mathrm{Br} \mathrm{J}$ Cancer. 2001;85(9):1335-9.

54. Kent WDT, Hall SF, Isotalo PA, Houlden RL, George RL, Groome PA. Increased incidence of differentiated thyroid carcinoma and detection of subclinical disease. CMAJ. 2007;177(11):1357-61.

55. How J, Tabah R. Explaining the increasing incidence of differentiated thyroid cancer. CMAJ. 2007;177(11):1383-4.

56. Butt P, Beirness D, Cesa F, Gliksman L, Paradis C, Stockwell T. L'alcool et la santé au Canada: résumé des données probantes et directives de consommation à faible risque. Ottawa (Ont.) : Centre canadien de lutte contre l'alcoolisme et les toxicomanies; 2011. 
57. Statistique Canada. Enquête sur la santé dans les collectivités canadiennes (ESCC). CANSIM - Tableau 105-0501 - Profil d'indicateurs de la santé, estimations annuelles, selon le groupe d'âge et le sexe, Canada, provinces et territoires, régions sociosanitaires (limites de 2011) et groupes de régions homologues, occasionnel [fichier de données]. Ottawa (Ont.) : Statistique Canada; [consulté le 19 sept. 2011]. Consultable à partir de la page: http://www5 . statcan.gc.ca/cansim/a05;jsessionid = ACE5E46E90BBE8567AB150991765AFBA? id $=1050501$ \& retrLang $=$ fra\&lang $=$ fra

58. Ahmad N, Flight J, Singh VA, Poole N, Dell CA. Enquête sur les toxicomanies au Canada (ETC) - Une enquête nationale sur la consommation d'alcool et d'autres drogues par les Canadiens - Tendances selon le sexe. Ottawa (Ont.) : Santé Canada; 2008.

59. Parkin DM. The global health burden of infection-associated cancers in the year 2002. Intl J Cancer. 2006;118(12):3030-44.

60. Chuang SC, La Vecchia C, Boffetta P. Liver cancer: descriptive epidemiology and risk factors other than HBV and HCV infection. Cancer Lett. 2009;286(1):9-14.

61. Solar and ultraviolet radiation.Lyon (FR) : International Agency for Research on Cancer; 1992. (IARC monographs on the evaluation of carcinogenic risks to humans 55)

62. Jemal A, Devesa SS, Hartge P, Tucker MA. Recent trends in cutaneous melanoma incidence among whites in the United States. J Natl Cancer Inst. 2001;93(9):67883.

63. Welch HG, Woloshin S, Schwartz LM. Skin biopsy rates and incidence of melanoma: population based ecological study. BMJ. 2005;331(7515):481.

64. The Ontario Sun Safety Working Group. Insight on cancer: sun exposure and protective behaviours in Ontario. An Ontario report based on the 2006 Second National Sun Survey. Toronto (Ont.) : Canadian Cancer Society; 2010 [consulté le 13 juin 2011]. Joint publication of Cancer Care Ontario. PDF (4,92 Mo) téléchargeable à partir du lien : http://uvnetwork.ca /InsightonCancerSunExposureand ProtectiveBehavioursinOntarioFinal.pdf
65. Joosse A, de Vries E, Eckel R, Nijsten T, Eggermont AM, Holzel D et collab. Munich Melanoma Group. Gender differences in melanoma survival : female patients have a decreased risk of metastasis. J Invest Dermatol. 2011;131(3):719-26.

66. Howlader N, Noone AM, Krapcho M, Neyman N, Aminou R, Waldron W, et collab. (dir.). SEER Cancer Statistics Review, 1975-2008. Bethesda (MD) : National Cancer Institute; 2011 [consulté le 13 décembre 2011]. Consultable à partir de la page : http://seer.cancer.gov/csr /1975_2008/

67. Morton DL. Current management of malignant melanoma. Ann Surg. 1990;212(2): 123-4.

68. Jayson M, Sanders H. Increased incidence of serendipitously discovered renal cell carcinoma. Urology. 1998;51(2):203-5.

69. Leslie JA, Prihoda T, Thompson IM. Serendipitous renal cell carcinoma in the post-CT era : continued evidence in improved outcomes. Urol Oncol. 2003; 21(1):39-44.

70. Chow WH, Gridley G, Fraumeni JF Jr, Jarvholm B. Obesity, hypertension, and the risk of kidney cancer in men. N Engl J Med. 2000;343(18):1305-11.

71. Pan SY, Johnson KC, Ugnat AM, Wen SW, Mao Y. Association of obesity and cancer risk in Canada. Am J Epidemiol. 2004; 159(3):259-68.

72. Howson CP, Hiyama T, Wynder EL. The decline in gastric cancer : epidemiology of an unplanned triumph. Epidemiol Rev. 1986;8(1):1-27.

73. Schistosomes, liver flukes and Helicobacter pylori. Lyon (FR) : International Agency for Research on Cancer; 1994. (IARC monographs on the evaluation of carcinogenic risks to humans 61)

74. Liu S, Semenciw R, Probert A, Mao Y. Cervical cancer in Canada : changing patterns in incidence and mortality. Int $\mathrm{J}$ Gynecol Cancer. 2001;11(1):24-31.

75. Comité consultatif national de l'immunisation. Déclaration sur le vaccin contre le virus du papillome humain. Relevé des maladies transmissibles au Canada 2007;33(DCC-2):1-32.
76. A review of human carcinogens: biological agents.Lyon (FR) : International Agency for Research on Cancer; 2012. (IARC monographs on the evaluation of carcinogenic risks to humans 100b)

77. Auluck A, Hislop G, Bajdik C, Poh C, Zhang $\mathrm{L}$, Rosin M. Trends in oropharyngeal and oral cavity cancer incidence of human papillomavirus (HPV)-related and HPVunrelated sites in a multicultural population: the British Columbia experience. Cancer. 2010;116(11):2635-44. 